\title{
EFICIENCIA PESQUERA DE LA NASA AMIGABLE, EN LAS CAPTURAS ARTESANALES DE LANGOSTA ESPINOSA (P. ARGUS) EN LOS CAYOS MISKITUS ${ }^{1}$
}

Jadder Mendoza-Lewis ${ }^{2}$

\begin{abstract}
RESUMEN
L a pesquería de langosta (Panulirus argus) en el caribe nicaragüense se encuentra en estado de depresión consecuencia de la incidencia de la mortalidad por pesca, principalmente por las altas capturas de organismos juveniles, por debajo de las tallas permitidas por la legislación nacional; ante este efecto negativo para la sobre vivencia del recursos y el rendimiento sostenible pesquero, se han impulsado diversos esfuerzos de protección biológica del recurso que van desde el establecimiento de tallas mínimas comerciales (Longitud mínima de cola de $140 \mathrm{~mm}$ y/o peso mínimo de cola de 5 onzas ó 140 gramos) y la veda temporal de capturas entre el 1 de abril y el 30 de junio; así también se ha promovido la prohibición de la pesca por buceo, la cual ha resultado de escasa aceptación por el rol económico de esta estrategia en la economía regional que genera empleo directo para más de 3000 buzos e igual número
\end{abstract} de cayuqueros.

Ante esta situación del recurso langosta del caribe ( $P$. argus) la Universidad de las Regiones Autónomas de la Costa Caribe Nicaragüense (URACCAN) y el Fondo Mundial para la Naturaleza (WWF) promueven la pesca sostenible en la Reserva Biológica Marina de los Cayos Miskitus, sitio estratégico para la biología del recurso mencionado y el desarrollo de la pesca artesanal, para lo cual se impulsa las capturas con nasas (trampas) que propicien la reducción de capturas de organismos con dimensiones por debajo de los parámetros pesqueros establecidos por la autoridad pesquera de Nicaragua.

1 Estudio desarrollado con financiamiento de USAID a través de WWF en el programa Centroamericano PROARCA APM.

2 Biólogo marino con experiencia en el manejo comunitarios de la Reserva Cayos Miskitus y los recursos naturales costeros. Director del Instituto de Recursos Naturales Medio Ambiente y Desarrollo Sostenible. E-mail Jadder.lewis@gmail.com. 
Con este fin durante el periodo de agosto 2005 a febrero del 2006 se promovió el ejercicio de experimentación pesquera de utilización de la Nasa Amigable (E1) en las capturas artesanales de langosta en los Cayos Miskitus, con el establecimiento de 180 nasas de tipo E1 y 180 nasas de tipo tradicional (tr) empleada en la pesquería mencionada, con el fin de evaluar la eficiencia pesquera de ambas artes de pesca, los parámetros biológicos de las capturas en ambos casos y determinar la contribución a la conservación del recursos por el arte alternativo, el proceso conllevó al levantamiento de información biométrica de las capturas de peso y tallas tanto a parámetros morfométricos como totales, cefalotórax y cola.

Los resultados obtenidos y presentados en este informe comprenden registros de las capturas en los meses de noviembre a febrero del la temporada 2005-2006 donde se obtienen referencia de importantes contribuciones de conservación en las capturas artesanales de langosta en los Cayos Miskitus; destacando las características de talla que permiten comparaciones con diferencias significativas; las capturas con la nasa tradicional (tr) alcanzan un 53\% de organismos por debajo e la talla legal; en tanto que las capturas con la Nasa Amigable E1 por debajo de $140 \mathrm{~mm}$ de longitud de cola representan el 31\% de las capturas lo que implica una reducción del $22 \%$ de organismos capturados que aún no alcanzan la talla legal de comercialización; así también se observa que las tallas promedios de las capturas son diferentes con medidas que son diferentes al estimar sus diferencias, aplicando una t-student obteniendo una talla promedio de $139.64 \mathrm{~mm}$. en tanto que empleando el arte alternativo esta se aumenta hasta $146.51 \mathrm{~mm}$.

\section{INTRODUCCIÓN}

La importancia socioeconómica de la langosta en las pesquerías de las zonas costeras de las regiones autónomas de Nicaragua y la relevancia que tiene para el manejo de los ecosistemas ha motivado un permanente interés en la gestión de manejo de la Reserva Biológica Marina Cayos Miskitus, que vincula como tema clave el control del acceso por las comunidades miskitas, siendo la principal fuente de recursos económicos comerciales.

No obstante la tendencia de las capturas reflejan un deterioro en la calidad del recurso biológico, que han ameritado el desarrollo de importantes estudios por la Administración de la Pesca y la Acuicultura (AdPESCA), que determinan una condición de plena explotación pesquera y que Ehrhadrt (2006), determina como una incidencia de la mortalidad por pesca de 2.4 veces mayor a la capacidad biológica aceptable; en este particular hay que agregar que en los últimos años la pesca artesanal ha aportado más del 
$50 \%$ de las capturas y en promedio organismos de menor tamaño y/o edad, considerando que son pesquerías en aguas más someras con respecto a la pesca industrial.

De manera que en la actualidad, y con la plataforma jurídica administrativa de la pesca que ofrece la ley 489 aprobada a finales del 2004, se definen diversos mecanismos de protección y desarrollo de la pesquería en mención, destacando (a) tallas mínimas comerciales, (b) veda temporal, (c) cuota global de captura anual y (d) regulación del esfuerzo con restricción de número de embarcaciones y artes de pesca, además destaca como (e) reducción y cierre de la pesca por buceo.

Por tanto esta ultima provoca el llamado a identificar medios de pesca u otras alternativas económicas para un gran número de empleos y que a su vez el potencial incremento de nasas venga a beneficiar la sobrevivencia del recurso; para lo cual el presente estudio ofrece los resultados de capturas de langosta en los Cayos Miskitus, empleando nasa de tipos (e1) diseñada por pescadores artesanales, con criterios que permitan su uso con el menor incremento de costos y contribuyan a la reducción de las capturas de organismos juveniles.

Con este fin se impulso las capturas de langosta en la pesquería artesanal, desarrollada en los Cayos Miskitus con la participación de 6 pescadores entre agosto 2005 y febrero 2006, determinándose que las capturas tradicionales se constituyen más del 50\% por organismos con tallas ilegales menores a $140 \mathrm{~mm}$ de longitud de cola, en tanto con la nasa del experimento estas sólo representan el 31\%, esta reducción superior al 20\% de capturas en organismos juveniles ofrece una importante contribución a la sostenibilidad del recurso y una alternativa de manejo de la pesca artesanal que beneficie en el mediano y largo plazo un crecimiento de las capturas legales.

\section{OBJETIVOS DEL ESTUDIO}

a) Evaluar el rendimiento pesquero y la factibilidad socioeconómica y bioecológica mediante la utilización de la Nasa Amigable seleccionada, en las pesquerías artesanales e industriales de langosta espinosa del caribe ( $P$. argus) en el caribe nicaragüense y establecer parámetros comparativos con la nasa tradicional utilizada en el área.

b) Seleccionar el diseño de nasa con espacios de escape más adecuado para la pesquería de langosta espinosa artesanal en la Moskitia Nicaragüense.

c) Determinar la eficiencia de captura de langosta espinosa ( $P$. argus) mediante la utilización de la nasa con rejilla de escape seleccionada como arte de pesca en la pesquería artesanal de Cayos Miskitus y compararla con la eficiencia de la nasa tradicional de madera actualmente utilizada en la pesca artesanal. 
d) Cuantificar el porcentaje de individuos por debajo de la talla mínima legal presente en las capturas de langosta con la nasa modelo y con la nasa de referencia.

e) Validar las equivalencias en las medidas de largo total, largo de tórax y cefalotórax y determinar el cambio en el cumplimiento de los estándares de calidad de los individuos medidos de diferentes formas.

\section{MARCO DE REFERENCIA DE LA PESQUERÍA DE LANGOSTA EN LOS CAYOS MISKITUS}

\subsection{Generalidades}

La pesquería de langosta se desarrolla en los Costa Caribe desde finales de los 50's y ha vendido siendo determinante en la economía de las zonas costeras en la Costa Atlántica de Nicaragua, donde siempre ha destacado el aporte a las capturas provenientes de pescadores artesanales y en esta última década la desarrollada por pescadores indígenas.

\subsection{El ambiente en Cayos Miskitus}

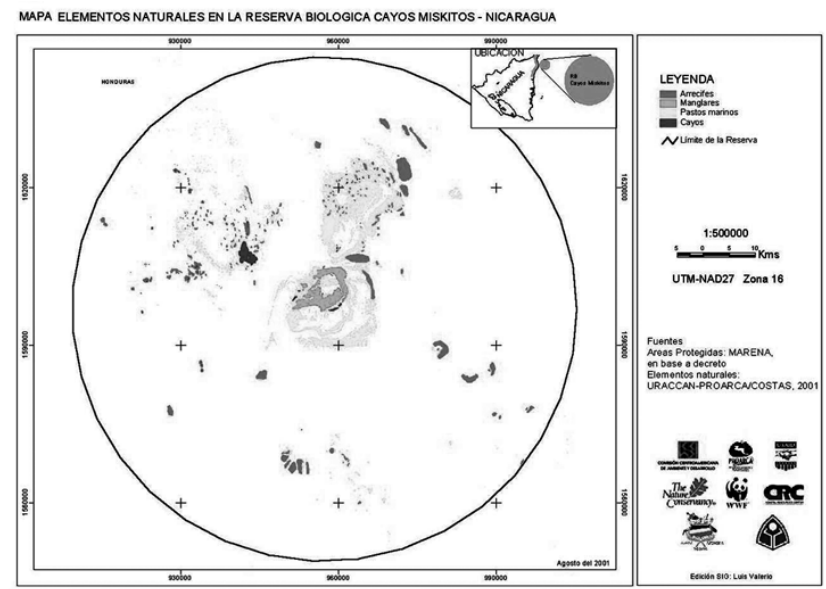

Los Cayos Miskitus representan un complejo ecosistema de alta diversidad de ambientes, marinos que asocia mangles, arrecifes coralinos, pastos marinos y jardines de octo corales, destaca en este ambiente que es de aguas someras que no supera los $40 \mathrm{~m}$ de profundidad (mapa).

La diversidad biológica es alta y ofrece un importante hábitat para el ciclo de vida de la langosta en su etapa, larvaria y de crecimiento juvenil; entre las principales amenazas señaladas por Fonseca. et. al. 2001, destaca el deterioro de los arrecifes por la alta incidencia del buceo para la pesca de langosta, así como la sedimentación proveniente del Rio Coco. 


\subsection{Dinámica socioeconómica de la Pesquería de langosta en los Cayos Miskitus}

La pesquería artesanal de langosta en los Cayos Miskitus es efectuada normalmente por pescadores indígenas Miskitos, que practican esta actividad históricamente a través del buceo principalmente libre (free dive), a finales de los 70's se inicio el ejerció del buceo autónomo (scuba) y después de los 80 's se fue estableciendo la pesca con nasas.

En la actualidad los desembarques de capturas comerciales de los Cayos Miskitus no se han establecido con exactitud, no obstante es la principal fuente de los registros de la pesca artesanal que para la temporada 2004-2005 represento el 51\% de un volumen de próximo a las 1,050 toneladas.

Como lo plantea Ehrhardt. (2006) el esfuerzo pesquero es menor (ver figura siguiente) donde la relación de pesca con nasas entre ambas pesquerías es significativa y

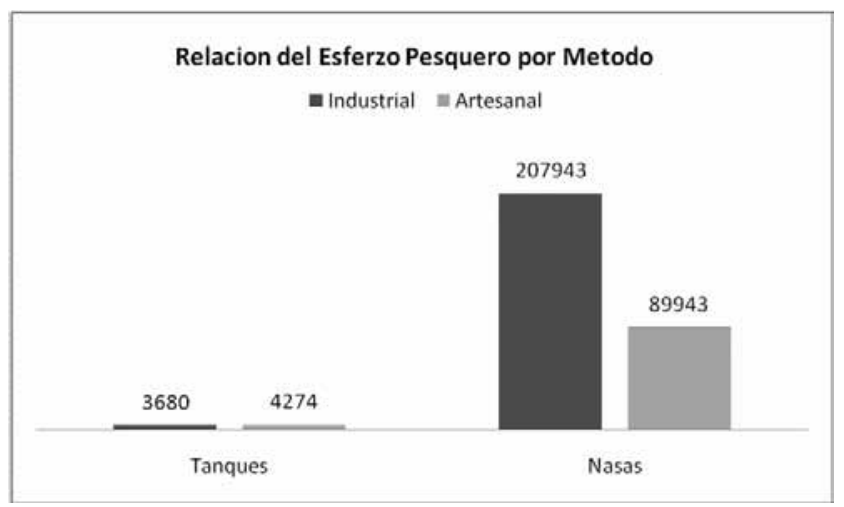
superior en la pesca industrial; se estima que esta estrategia de pesca artesanal es una principal actividad económica en Cayos Miskitus, cabe señalar que la pesca a travez del buceo es importante y superior a la de nasa, no obstante la política de cierre de esta forma de pesca, crea una mayor presión a las capturas con nasas.

\section{ENFOQUE METODOLÓGICO}

El enfoque metodológico del estudio se basa en un análisis comparativo de las capturas en talla y volúmenes entre las nasas de uso actual por los pescadores artesanales de los Cayos Miskitus y la nasa $\mathrm{E}_{1}$ propuesta por el proyecto con el propósito de establecer una alternativa tecnológica que induzca en la reducción de las capturas de langostas P. argus de tallas menores a la establecida por el marco legal de Nicaragua.

De manera que el estudio permitirá conocer la eficiencia pesquera de la nasa $E_{1}$ en comparación con las nasas tradicionales de uso actual por las comunidades de pescadores artesanales de langosta, el proceso de investigación incluirá registros de variables biológicas, ecológicas y económicas que inciden en el esfuerzo pesquero, llevando un análisis de carácter monovariado y multivariado. 


\subsection{LAS UNIDADES DE MUESTRA Y TIPOS DE MUESTRAS}

El análisis comparativo partirá preestableciendo como unidades de muestra la nasa, a fin de conocer la eficiencia de captura de las nasas según sus características ${ }^{3}$ técnica las cuales principalmente se agruparan por dimensiones cúbicas de la nasa y la abertura de las rejillas de escape, siendo que este ultimo factor incide en las tallas mínimas de las capturas por el esfuerzo pesquero.

Se han preestablecido 2 tipos $^{4}$ de muestra según el tipo de nasa, agrupada por los siguientes criterios:

\begin{tabular}{|lccccc|}
\multicolumn{1}{c}{ Tipo de nasa } & $\begin{array}{c}\text { Ancho } \\
\text { (pulgadas) }\end{array}$ & $\begin{array}{c}\text { Largo } \\
\text { (pulgadas) }\end{array}$ & $\begin{array}{c}\text { Altura } \\
\text { (pulgadas) }\end{array}$ & $\begin{array}{c}\text { Abertura } \\
\text { de Rejilla }\end{array}$ & $\begin{array}{c}\text { Matadero } \\
\text { (pulgadas) }\end{array}$ \\
\hline $\begin{array}{l}\text { Nasa tradicional } \\
\begin{array}{l}\text { (utilizadas en el } \\
\text { experimento) }\end{array}\end{array}$ & 24 & 38 & 14 & 1.5 & $5 * 5$ \\
Nasa Amigable E1 & 24 & 38 & 14 & 2.25 & $7.5 * 7.5$ \\
\hline
\end{tabular}

\subsection{TAMAÑO DE LA MUESTRA}

El tamaño de las muestras se estableció en 360 nasas con 180 de tipo $\mathrm{E}_{1}$ y 180 de nasas tradicionales lo que representa el universo total de este tipo de arte de pesca.

\subsection{Tipos de nasas (tratamiento y estratos)}

El diseño se fundamenta en el empleo de dos condiciones basada en el tipo de nasa, lo cual permite determinar 2 tratamientos 1) Nasas Tradicionales (testigo) y 2) Nasas Experimentales $\mathrm{E}_{1}$. El acomodo de las nasas se establece en forma intercalada en lingadas de 10 nasas contando cada lingada con 5 nasas de cada tipo, esto permitirá capturas bajo un mismo valor probabilístico de capturas considerando el principio de espacio, profundidad y condiciones hidrológicas.

\subsection{Variables de Medición}

El estudio demanda del registro de datos tanto biológico, pesquero y económico a fin de poder conocer el comportamiento de la pesquería artesanal de langosta con nasas en los

3 Las características técnicas de la nasa principalmente se establecen en el volumen total de la nasa (ancho, largo y altura), abertura de la rejilla de escape y tamaño del orificio de penetración (matadero).

4 Los tipos de nasas provienen de referencias de reunión con pescadores artesanales y sistematizados por Jonny Maradiaga el 12 de agosto del 2005. 
Cayos Miskitus tanto desde la perspectiva biológica del recurso, como desde los costos de producción que cubren los pescadores.

\subsection{Procesamiento estadístico de datos}

Las pruebas estadísticas se fundamentan en un análisis de tendencia central entre ambos estratos para estimar las características morfométricas de talla y peso en las capturas de ambas artes de pesca (nasas) involucradas en el experimento, para dicho análisis se emplearan datos morfométricos de talla total y talla de cola medidas en $(\mathrm{mm})$, a igual se emplean datos de peso tanto total como de cola ambos medidos en (gr).

\section{HIPÓTESIS DEL ESTUDIO}

Ho: Las tallas promedio de las capturas de langostas espinosa (Panulirus argus) con las nasas tradicionales son iguales a las tallas promedio de las capturas con la nasa experimental $\mathrm{E}_{1}$.

$$
\text { Ho: } \mathrm{L}(\mathrm{tr})=\mathrm{L}\left(\mathrm{e}_{1}\right)
$$

Hi: Las tallas promedio de las capturas de langosta espinosa (Panulirus argus) con las nasas tradicionales son diferentes a las tallas promedio de las capturas con la nasa experimental $\mathrm{E}_{1}$.

$$
\text { Hi: } \mathrm{L}(\mathrm{tr}) \neq \mathrm{L}\left(\mathrm{e}_{1}\right)
$$

Estimación de las medidas de tendencia central de las capturas en tallas

Las tallas de las capturas se lograrán mediante la medición de cada organismo, las cuales se registraran en una base de datos según su tratamiento sea este perteneciente a nasas tradicionales o nasas experimentales, para estimar:

- Las tallas promedio de las capturas por tipo de nasa.

- La varianza y desviación estándar de cada captura por tipo de nasa.

Esto contribuirá a un análisis de varianza para estimar si las variaciones de las tallas medias son iguales estadísticamente o si las variaciones son significativas, el análisis de varianza se realizara utilizando una prueba de t-student considerando que el comportamiento es paramétrico y que sigue una distribución normal. 


\section{RESULTADOS}

\subsection{Generalidades}

Los resultados del estudio de estimación de la eficiencia pesquera de la Nasa Amigable $\mathrm{E}_{1}$ corresponden al análisis matemático y estadístico de datos biológico pesqueros levantados en los caladeros de pesca adyacente a los Cayos Miskitus y representan las capturas registradas en los meses de Noviembre- Diciembre del 2005 así como de Enero y parte de Febrero del año 2006, tanto en las capturas registradas de 180 Nasas Amigables $\mathrm{E}_{1}$ como en 180 nasas tradicionales que se establecieron para el experimento.

Para fines de este estudio se partió de la definición de un arte de pesca diseñado por pescadores artesanales de los Cayos Miskitus que sea rentable desde el enfoque pesquero e induzca a la conservación del recurso langosta en los Cayos Miskitus. Posteriormente se registraron las capturas de ambos tipos de nasas basado en la matriz establecida en el método y técnica de muestreo.

Un aspecto importante de los resultados es que a pesar que el esfuerzo de construcción de Nasas Amigables fue de 180 nasas, se concentró el registro en las capturas de 3 pescadores que brindaron series de datos a lo largo de 3 meses de 90 nasas tanto de tipo $\mathrm{E}_{1}$ como Tradicionales; los otros 3 pescadores no desarrollaron temporadas regulares aduciendo una temporada con alta frecuencia de nortes.

\subsection{LA NASA AMIGABLE E1 (CARACTERÍSTICAS Y CRITERIOS TÉCNICAS)}

La nasa amigable $\mathrm{E}_{1}$ es el resultado de un trabajo conjunto de pescadores artesanales de langosta en los Cayos Miskitus con el apoyo de expertos técnicos que han desarrollado experiencia en el área de estudio, y que se diseñó fundamentado en criterios tanto pesqueros como económicos y de viabilidad social de aceptación por los usuarios potenciales para lo cual destacaron como elementos claves:

a) Facilidad de operación por las capacidades instaladas de la pesquería.

b) Construirla con materiales disponibles y accesibles a los pescadores artesanales.

c) Que no representara mayores costos de inversión del capital instalado en la pesquería.

d) Adoptar el mayor ajuste a las normas técnicas oficiales para las pesquerías de Nicaragua.

e) Contribuyan a la reducción de las capturas de organismos menores a las tallas permitidas por las leyes nacionales. 
f) Fácil degradación en el ambiente para evitar capturas en periodos de tiempos prolongados (Caso de extravíos).

Lo cual condujo a la definición de la nasa la cual mantiene dimensiones volumétricas cercanas a las tradicionales que se emplean el área de estudio por los pescadores artesanales y que brinde mayor probabilidad de escape de juveniles estableciéndose las siguientes especificaciones técnicas:

Nasa de madera por su facilidad de adquisición, mantenimiento en caladeros de pesca y resistencia de hasta 2 ańos ( 2 temporadas de pesca) a las condiciones ambientales marinas, teniendo de preferencia la construcción con nanciton (Hyeronyma alchorneoides) que es un recurso forestal abundante y factible de aprovechar con planes de manejo, no esta sometido al proyecto de moratoria forestal que impulsa en gobierno de Nicaragua (ver foto de nasa).

Con dimensiones de una altura de 14 pulgadas, ancho de 28 pulgada y el largo de 40 pulgadas, que permitan su fácil utilización y manipulación en las faenas de pesca, considerando que es el esfuerzo humano el que se emplea para las jaladas.

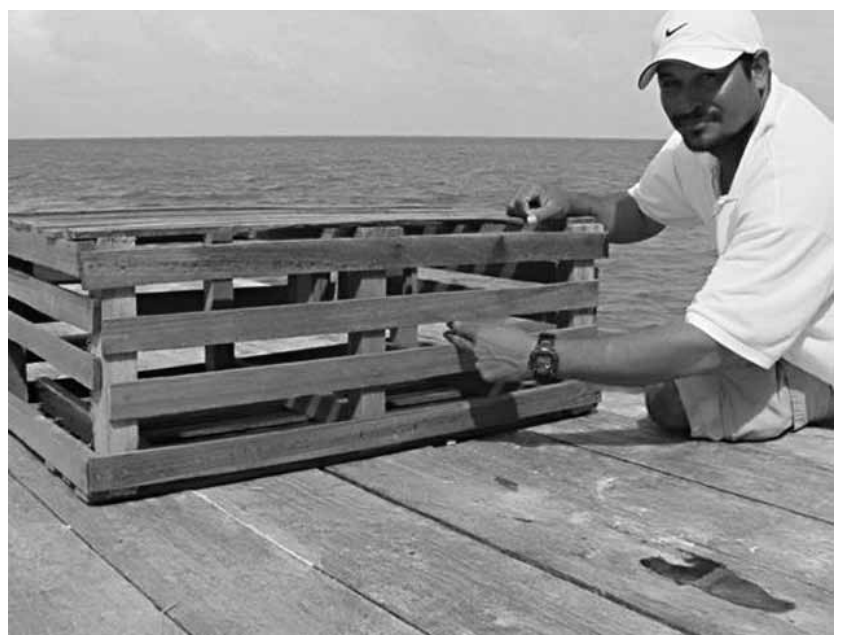

Con aberturas de escapes de 2.25 pulgadas que permita la salida de organismos de dimensiones menores a las establecidas por la ley 489 que establece como talla mínima de captura de $140 \mathrm{~mm}$ de longitud de cola.

Los costos estimados de las nasas son de \$23.49 dólares americanos por cada unidad incluyendo los insumos, materiales, mano de obra de su construcción y el mecate (soga) para las lingadas que normalmente contienen de 10 a 12 nasas a una distancia entre nasas de 16 brazadas ( $24 \mathrm{~m}$ ).

Tabla 1. Costos de la Nasa E1

\begin{tabular}{|cccccc|}
\hline Insumo & Cantidad & $\begin{array}{c}\text { Unidad de } \\
\text { Medida }\end{array}$ & $\begin{array}{c}\text { Costo } \\
\text { Unitario }\end{array}$ & $\begin{array}{c}\text { Subtotal } \\
\text { C\$. }\end{array}$ & Subtotal \$ \\
\hline Madera & 18 & Pies & 8 & 144,00 & 8,52 \\
$\begin{array}{c}\text { Clavos } \\
\text { Galvanizados }\end{array}$ & 1 & Libra & 25 & 25,00 & 1,48 \\
\hline
\end{tabular}




\begin{tabular}{|cccccc|} 
Insumo & Cantidad & $\begin{array}{c}\text { Unidad de } \\
\text { Medida }\end{array}$ & $\begin{array}{c}\text { Costo } \\
\text { Unitario }\end{array}$ & $\begin{array}{c}\text { Subtotal } \\
\text { C\$. }\end{array}$ & Subtotal \$ \\
\hline $\begin{array}{c}\text { Cemento } \\
\text { Cortado de }\end{array}$ & 0,2 & Saco & 140 & 28,00 & 1,66 \\
Madera & 18 & Pies & 1,5 & 27,00 & 1,60 \\
Mano de Obra & 1 & Nasa & 18 & 18,00 & 1,07 \\
Mecate & 0,1 & Rollo $34^{\prime \prime}$ & 1400 & 140,00 & 8,28 \\
Bollas & 0.2 & Boyas & 80 & 16,00 & 0,88 \\
\hline \multicolumn{7}{r}{ Totales } & & 398.00 & 23,49 \\
\hline
\end{tabular}

Este costo de construcción de nasa no incluye otras variables de costo como el valor del transporte acuático del material desde Bilwi o cualquier comunidad a los Cayos Miskitus, así como los costos de transporte de los campamentos pesqueros hacia los caladeros, estos costos dependen de la estrategia de cada pescador debido a que se han identificado 2 formas la primera que consiste en llevar los materiales e insumos desarmados y construir las nasas en el campamento pesquero y la segunda se fundamenta en llevar las nasas armadas desde Bilwi a los Cayos Miskitus.

Un aspecto importante es que la nasa amigable $\mathrm{E}_{1}$ se recomienda con materiales orgánicos que se degradan rápidamente si no se le brinda el mantenimiento debido que consiste fundamentalmente en lavarlas y quitarles acumulación de sedimentos marinos que se depositan sobre ella, así también cambiar reglar que han sido dañadas por peces u otros organismos, de acuerdo a la experiencia de los pescadores cada faena debe tener un tiempo máximo de calado no mayor de 12 días para asegurar un buen cuido del arte de pesca.

\subsection{Comportamiento de las capturas}

Las capturas de las faenas registradas son muy variables y obedecen a las condiciones climáticas, para el período del estudio se observo que el tiempo de calado por faena fue irregular y se obtuvo un comportamiento normal de mayores capturas a inicios de la temporada con organismos de mayor tamańo y una reducción drástica para los meses de enero y febrero del 2006. En el análisis comparativo de las captura por tipo de nasa empleada en el experimento determino que los volúmenes de las capturas son superiores con la nasa (Tr) alcanzando volúmenes de 6.07 libras de colas comerciales con el calado 151 nasas (tr) en tanto que las capturas de 151 nasas $\mathrm{E}_{1}$ promediaron capturas de 3.01 libras lo cual es menor del $50 \%$ en cuanto a volumen se refiere.

Sin embargo un aspecto relevante de la nasa $\mathrm{E}_{1}$ es un aumento en los volúmenes de capturas con el aumento de este tipo de nasa y que este se mantiene estable o es menor con la nasa (tr). 
Como se puede apreciar en los gráficos de regresión la incidencia del número de nasas de tipo $\mathrm{E}_{1}$ tiene una mayor ingerencia en las capturas en tanto que la nasa (tr) tiene una menor incidencia en las capturas, esto es más claro en el análisis de la estructura de tallas, donde se observa la alta incidencia de organismos menores de $140 \mathrm{~mm}$ de longitud de cola que son capturados por la nasa (tr). Este aspecto también se resalta en el nivel de correlación entre el numero de nasas y las capturas teniendo para $\mathrm{E}_{1}(\mathrm{r}=0.4389)$ en tanto que para tr $(\mathrm{r}=0.1164)$, demostrándose un mayor efecto de capturas crecientes por la nasa de tipo $\mathrm{E}_{1}$.
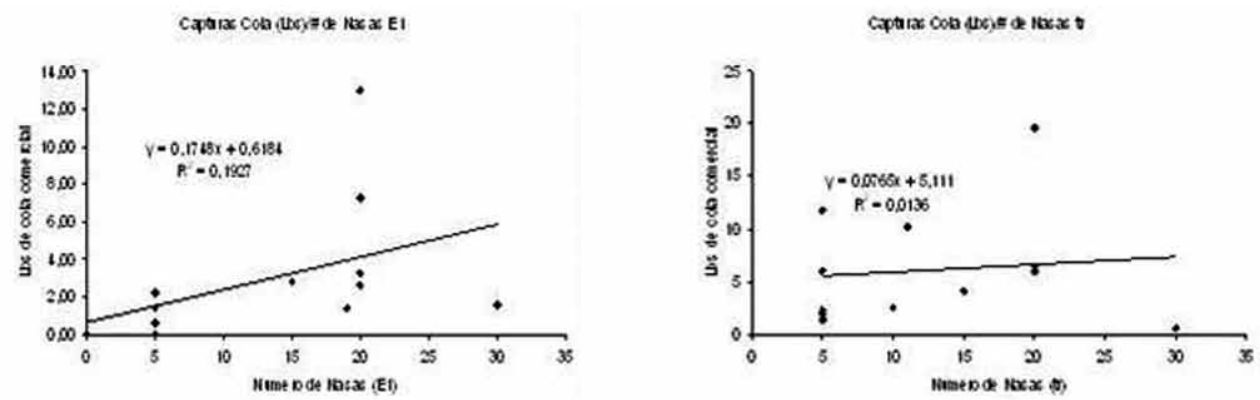

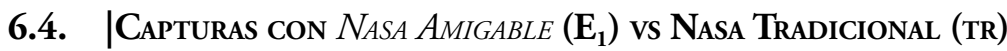

El análisis de las capturas entre ambos tipos de nasas en los Cayos Miskitus para la captura de langosta se fundamenta en comparar la estructura de tallas que componen las capturas por cada tipo de nasa considerando variable morfométricas como las medidas de longitud (total, cefalotórax y cola) así como el peso total y de cola del organismo.

Esto condujo a un análisis de resultados de las capturas que compara la eficiencia de pesca o selectividad en ambas jaulas obteniendo:

\section{Capturas totales vs. capturas liberadas}

Para el análisis de organismos liberados se consideran las capturas de los individuos que al levantar las nasas se encuentran atrapados y que su tamaño es menor que el aprovechado para la comercialización y que en la pesca responsable deben ser liberados, este fenómeno de liberación se ha venido incrementando, no obstante no es practica regular y en las nasas tradicionales los pescadores emplean estos organismos pequeños para llamado para mejorar las capturas por nasa.

Las capturas de langosta por tipo de nasa presenta resultados bien diferenciados como consecuencia de una mayor selectividad de organismos mayor tamańo en las nasas amigables para el caso de las capturas y liberaciones de langostas por debajo de longitudes de cola de los $100 \mathrm{~mm}$ se obtuvo una incidencia mayor por la nasa tradicional que capturo en 62 ocasiones y solamente en 38 ocasiones por la nasa $\mathrm{E}_{1}$ con capturas promedio 
de 6.61 organismo para la nasa (tr) superior a 4.68 organismos atrapados con la nasa $E_{1}$ estas diferencias de medias de la captura resultan diferentes significativamente de acuerdo a una comparación de medias utilizando pruebas de t-student.

Tabala 2. Número de organismos Capturados por tipo de Nasa

One-Sample Statistics

\begin{tabular}{|lcccc|} 
& N & Mean & Std. Deviation & $\begin{array}{c}\text { Std. Error } \\
\text { Mean }\end{array}$ \\
\hline Capturas totales $\left(\mathrm{E}_{1}\right)$ & 38 & 4,6842 & 2,85795 &, 46362 \\
Capturas Totales $(\mathrm{tr})$ & 62 & 6,6129 & 4,33951 &, 55112 \\
\hline
\end{tabular}

De acuerdo al análisis de varianza de las medias de las capturas en número de organismo por tipo de nasa se observa que estas son diferentes significativamente con ligero nivel de traslape; sin embargo, en el siguiente inciso se analizará la estructura de las capturas donde se identifica una alta incidencia de organismos pequeños por debajo de las tallas comerciales permitidas por la ley.

Tabla 3. Análisis de varianza de las capturas promedio en numero de organismo por tipo de nasa

One-Sample Test, Test Value $=0$

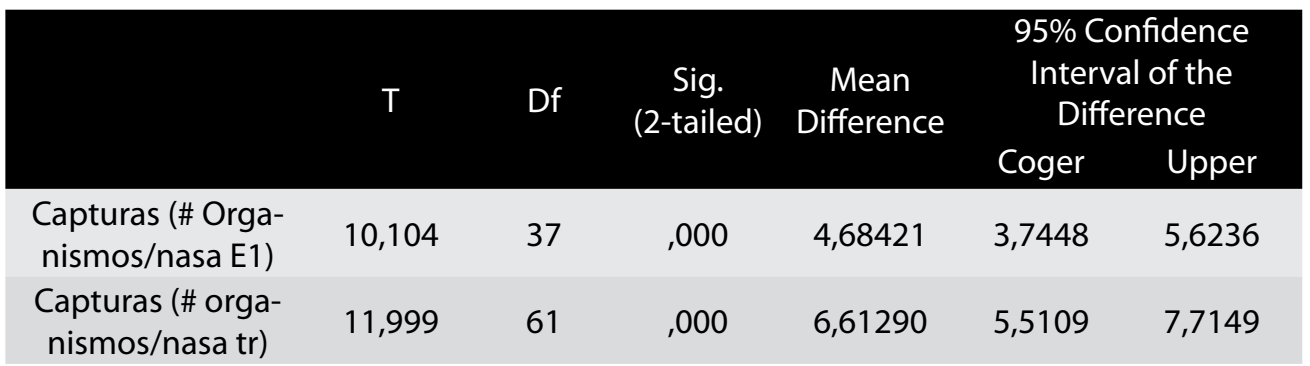

Así también en la relación de organismos que no alcanzan la longitud de cola de $100 \mathrm{~mm}$ se observa que se liberan 2.18 organismo en el caso de la nasa $\mathrm{E}_{1}$ lo que representa el $46.4 \%$ del total de las capturas en tanto que la nasa tradicional captura 3.29 organismos por debajo de la talla de cola mencionada que representa el $49.75 \%$. 
Tabla 4. Promedios de Organismos liberados por tipo de Nasa

One-Sample Statistics

\begin{tabular}{|ccccc|} 
& N & Mean & Std. Deviation & $\begin{array}{c}\text { Std. Error } \\
\text { Mean }\end{array}$ \\
\hline $\begin{array}{c}\text { Capturas liberadas/ } \\
\text { nasa } E_{1}\end{array}$ & 38 & 2,1842 & 1,70619 &, 27678 \\
$\begin{array}{c}\text { Capturas liberadas/ } \\
\text { nasa Tr }\end{array}$ & 62 & 3,2903 & 2,70632 &, 34370 \\
\hline
\end{tabular}

Estas variaciones de medias en el número de organismos liberados por el tipo de nasa de igual manera que en las capturas que fueron liberadas.

Tabla 5. Análisis de Varianza de capturas liberadas por tipo de nasa

One-Sample Test, Test Value $=0$

\begin{tabular}{|ccccccc|} 
& t & Df & Sig. (2-tailed) & $\begin{array}{c}\text { Mean } \\
\text { Difference }\end{array}$ & $\begin{array}{c}95 \% \text { Confidence Inter- } \\
\text { val of the Difference } \\
\text { Coger }\end{array}$ & Upper \\
\hline $\begin{array}{c}\text { Capturas libera- } \\
\text { das/ nasa E }\end{array}$ & 7,891 & 37 &, 000 & 2,18421 & 1,6234 & 2,7450 \\
$\begin{array}{c}\text { Capturas libera- } \\
\text { das/ nasa Tr }\end{array}$ & 9,573 & 61 &, 000 & 3,29032 & 2,6030 & 3,9776 \\
\hline
\end{tabular}

Otro análisis relevante es una prueba de regresión que permite ver la incidencia de las capturas por cada tipo de nasa con la liberación de organismos por debajo de los 100 $\mathrm{mm}$ de longitud de cola, se observa que la probabilidad de liberar un organismo empleando la nasa $\mathrm{E}_{1}$ es del $35 \%$ lo cual representa aun una alta incidencia sobre organismos juveniles.

Capturas vs Liberados con Nasa (E1)

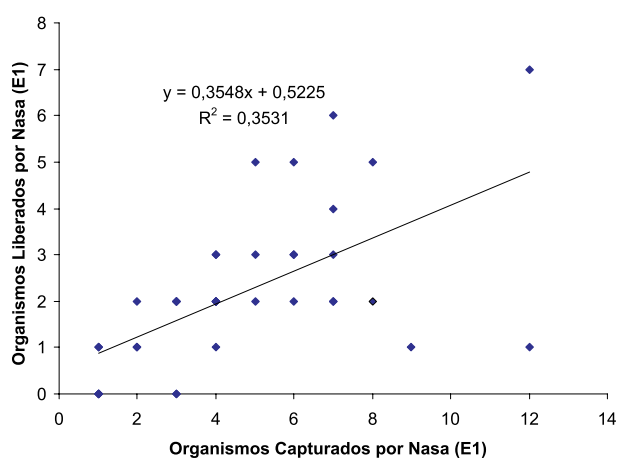

Capturas vs Liberados con Nasa (Tr)

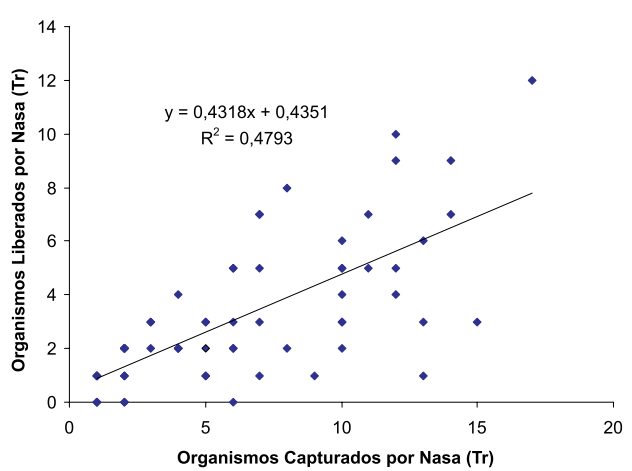


En tanto que la relación de capturas y liberaciones con la nasa tradicional es $47 \%$ probable que se capture un individuo por debajo de la talla aprovechable por el comercio, destacando que en el caso de la nasa $\mathrm{E}_{1}$ la probabilidad se reduce al $35 \%$. En el análisis de la estructura por talla se observaran los detalles de incidencia por tipo de nasa con organismos que presentan longitudes de cola mayores a los $100 \mathrm{~mm}$ e inferiores a los $140 \mathrm{~mm}$ esta ultima dimensión en la talla mínima comercial que establece la legislación nacional.

\section{Estructura de tallas capturadas}

Las capturas comerciales seleccionadas por los pescadores oscilaron entre los $110 \mathrm{~mm}$ $230 \mathrm{~mm}$ de longitud de cola siendo el rango para la nasa (tr) de $120 \mathrm{~mm}$ en tanto que para la nasa $E_{1}$ la selectividad fue mayor con un rango de captura comercial entre los $120 \mathrm{~mm}$ $190 \mathrm{~mm}$. La muestra para el análisis de la tallas de captura provienen de 181 organismos capturados con el método tradicional y 99 organismos capturados con la nasa $\mathrm{E}_{1}$.

Las capturas en talla (longitud de cola $\mathrm{mm}$ ) de langosta con la nasa tradicional (tr) fueron de 181 organismos seleccionados para el comercio en 151 nasas que experimentaron capturas mas la liberación de 204 organismos. Las capturas en tallas para la nasa (tr) presentan una media de $139.64 \mathrm{~mm}$ con una desviación estándar de 14.15; en tanto que las capturas en talla promedio con la nasa $\mathrm{E}_{1}$ ascendió hasta los $146.51 \mathrm{~mm}$ con desviación estándar de 15.68 .

\begin{tabular}{|c|c|c|c|}
\hline \multicolumn{2}{|c|}{$\begin{array}{c}\text { Tendencia central de medidas de peso } \\
\text { de cola }(\mathrm{mm}) \\
\text { Statistics }\end{array}$} & $\begin{array}{l}\text { Longitud de Cola } \\
\qquad \mathrm{mm}(\mathrm{E} 1)\end{array}$ & $\begin{array}{l}\text { Longitud de Cola mm } \\
\text { (Tr) }\end{array}$ \\
\hline $\mathrm{N}$ & Valid & 99 & 181 \\
\hline & Missing & 82 & 0 \\
\hline Mean & & 146,5152 & 139,6464 \\
\hline Std. Deviation & & 15,68788 & 14,14947 \\
\hline Variante & & 246,109 & 200,208 \\
\hline Range & & 75,00 & 90,00 \\
\hline
\end{tabular}

Como podemos apreciar en el anexo 3 y 4 las frecuencias acumuladas las capturas con la nasa (tr) por debajo de los $140 \mathrm{~mm}$ de longitud de cola representan $53 \%$ de las capturas en tanto que las capturas con nasa $\mathrm{E}_{1}$ por debajo de los $140 \mathrm{~mm}$ de longitud de cola solo representan el $31.3 \%$ lo que permite asumir una reducción considerable de organismos juveniles empleando el arte alternativo. 

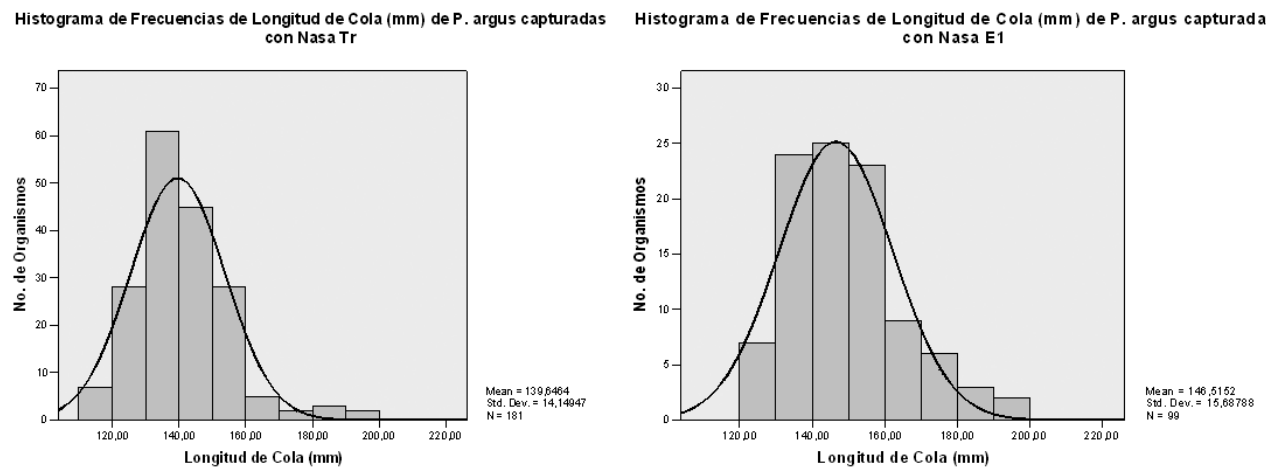

Otro aspecto relevante en el análisis de las capturas de tallas en peso (ver anexo 5 y 6) es la representatividad del $59.1 \%$ de capturas por debajo de las 5 onzas que establece la ley empleando la nasa (tr) en tanto que las capturas de organismos con peso de cola por debajo de las 5 onzas empleando la nasa $\mathrm{E}_{1}$ solo representan el $38.4 \%$.

Análisis de varianza de las capturas

Como se definió en los objetivos del estudio y las necesidades de validar las diferencias de las tallas de capturas en ambos tipos de nasa se efectuaron análisis de varianza (anova) con 95\% de confiabilidad empleando prueba de t-student para muestras de diferentes tamaños (n) obteniéndose como resultado que las medias en talla no son iguales, aceptando la hipótesis alternativa $\mathrm{H}_{1}$ : Longitud media de cola $(\mathrm{tr}) \neq$ Longitud media de cola $\mathrm{E}_{1}$.

One-Sample Test, Test Value $=0$

\begin{tabular}{|c|c|c|c|c|c|c|}
\hline & \multirow[t]{2}{*}{$\mathrm{t}$} & \multirow{2}{*}{ Df } & \multirow{2}{*}{$\begin{array}{c}\text { Sig. } \\
\text { (2-tailed) }\end{array}$} & \multirow{2}{*}{$\begin{array}{c}\text { Mean } \\
\text { Difference }\end{array}$} & \multicolumn{2}{|c|}{$\begin{array}{l}95 \% \text { Confidence Inter- } \\
\text { val of the Difference }\end{array}$} \\
\hline & & & & & Coger & Upper \\
\hline $\begin{array}{c}\text { Talla Cola }(\mathrm{mm}) \\
\text { E1 }\end{array}$ & 92,926 & 98 & ,000 & 146,51515 & 143,3863 & 149,6440 \\
\hline $\begin{array}{c}\text { Talla Cola }(\mathrm{mm}) \\
\mathrm{Tr}\end{array}$ & 132,779 & 180 & ,000 & 139,64641 & 137,5711 & 141,7217 \\
\hline
\end{tabular}

Considerando que intervalo de confianza superior de la media de las capturas en talla con (tr) es de 141.72 en tanto que el intervalo inferior de con $\mathrm{E}_{1}$ es de 143.38 con un a de 0.05 . 
Este comportamiento de diferencias de media en las capturas por ambos tipos de nasas es mas notable en el análisis de peso de cola teniendo que la media de peso (gr) capturadas con la nasa (tr) es 135.02 gr con un limite de confianza de $95 \%$ que esta es diferente al peso promedio de cola (gr) de las capturas empleando la nasa $\mathrm{E}_{1}$ que es de $153.63 \mathrm{gr}$.

One-Sample Test, Test Value $=0$

\begin{tabular}{|c|c|c|c|c|c|c|}
\hline & \multirow[t]{2}{*}{$\mathrm{t}$} & \multirow[t]{2}{*}{ Df } & \multirow[t]{2}{*}{$\begin{array}{c}\text { Sig. } \\
\text { (2-tailed) }\end{array}$} & \multirow[t]{2}{*}{$\begin{array}{c}\text { Mean } \\
\text { Difference }\end{array}$} & \multicolumn{2}{|c|}{$\begin{array}{l}\text { 95\% Confidence } \\
\text { Interval of the } \\
\text { Difference }\end{array}$} \\
\hline & & & & & Lower & Upper \\
\hline $\begin{array}{l}\text { Peso cola (gr) cap- } \\
\text { turados con } E_{1}\end{array}$ & 33,967 & 98 & ,000 & 153,63636 & 144,6603 & 162,6124 \\
\hline $\begin{array}{l}\text { Peso cola (gr) cap- } \\
\text { turados con (tr) }\end{array}$ & 29,432 & 180 & ,000 & 135,02762 & 125,9749 & 144,0803 \\
\hline
\end{tabular}

De manera que un aspecto relevante a considerar es la relación de la estructura en peso de las capturas considerando que el aporte de juveniles en longitud de cola es de $53 \%$ empleando la nasa de tipo (tr) en tanto que estos mismo organismos representan en peso el $59.1 \%$ lo cual es significante en términos de mortalidad por pesca con el arte tradicional.

\section{VII.DISCUSIÓN}

Evidentemente las referencias de las capturas de juveniles de langosta ( $P$. argus) en la pesquería del caribe Nicaragüense en múltiples estudios de la administración de la pesca desarrollado por Barnutty (2002 y 2000) se refleja la reducción en las tallas de las capturas, principalmente en los últimos 5 años posterior al auge de capturas del año 2000 que la pesquería aporto un volumen de 2 millones de libras.

De acuerdo con el análisis bioeconómico de esta pesquería desarrollado por Ehrhar$\mathrm{dt},(2005)$ esta pesquería presenta una alta incidencia en juveniles que en totalidad tanto de las capturas industriales de aguas mas profundas con respecto a las capturas artesanales adyacentes a arrecifes coralinos representan un estado critico de desembarques de tallas ilegales del $40 \%$, de manera que una primera aproximación a las capturas de la pesquería artesanal de los Cayos Miskitus conduce a asumir que esta aporta el 53\% para la parte media de la temporada, previendo que para los meses de febrero y marzo esta incidencia es mayor (Pescadores de Cayos Miskitus, 2005). 
No obstante empleando la nasa tradicional la reducción del aporte de juveniles puede limitarse al 31\% lo cual representa un aporte sustancial a la sobre vivencia del recurso es etapas juveniles y una reducción en la mortalidad por pesca ( $f$ ) que para al ańo 2004 Barnutty estimo en 0.5/año, considerando que este mismo autor refleja que el aporte a los desembarque de la flota artesanal a aumentado hasta el 51\%, es pertinente considerar el aporte concreto en sobrevivencia que brinda la nasa $\mathrm{E}_{1}$ a la captura sostenible del recurso langosta en los Cayos Miskitus.

Es evidente que la adopción de la nasa amigable $\mathrm{E}_{1}$ genera una recuperación de la población, no obstante actualmente las capturas de juveniles que representan el $60 \%$ de las capturas con la nasa tradicional representan importante ingresos comercializando este recurso por debajo de $\$ 3.00 / \mathrm{Lb}$, de manera que una transformación de la nasas tradicional a amigables requiere de un incremento en el esfuerzo pesquero, teniendo presente que la correlación de numero de nasas por capturas fue de $(r=0.43)$ en tanto que con el uso de $\operatorname{tr}(\mathrm{r}=0.11)$

Otro aspecto relevante es el efecto retomado de (Ehrhardt 2005) es la mortalidad por pesca en las edades de 2 y 3 años para la nasa tr en tanto que la nasa $\mathrm{E}_{1}$ tiene mayor efecto sobre las edades 3 y 4 lo cual de acuerdo a datos de precio por tamaño del recurso se estima que las capturas comerciales legales tienen precios de $\$ 12.37-\$ 12.74$ con la nasa tradicional; en tanto que las capturas con la nasa $\mathrm{E}_{1}$ tienden a comercializarse entre $\$ 12.74-\$ 13.63$ por libra, de manera que un aumento promedio de las capturas en una edad superior podría generar un ingreso mayor de $\$ 1.00$ por cada libra capturada, además que aproximadamente el $70 \%$ de las capturas con peso promedio de cola superior a las 5 onzas.

Un análisis a considerar sobre la estimación de organismos liberados es la limitada conciencia de los pescadores de liberar este grupo de organismos, por lo tanto la captura empleando la nasa $\mathrm{E}_{1}$ reduce considerablemente esta captura, lo cual en el fomento de nasas amigables asegura una mayor conciencia en la pesca, esta transformación debe darse en un proceso paulatino reduciendo la cantidad de nasas tradicionales y adoptando las aberturas de las nasa amigable para asegurar el escape de organismos juveniles.

\section{CONCLUSIONES}

Las capturas de langosta (P.argus) en los Cayos Miskitus empleando las nasas tradicionales tienen una alta incidencia en la mortalidad por pesca en organismos juveniles por debajo del los $140 \mathrm{~mm}$ de longitud de cola que alcanzan hasta mas del 50\% de las capturas, esto ha venido incidiendo dramáticamente en la reducción del recursos a escala global que demuestra tendencias anuales negativas como resultado de una sobre pesca al crecimiento de la población, especialmente por la pesca artesanal que ha incrementado su importancia pesquera hasta el $51 \%$ de los desembarques nacionales (Barnutty, 2004). De manera que 
urgente atender medidas de protección y uso sostenible del recurso que reduzcan la mortalidad en juveniles que aporten a las medidas de sostenibilidad que se impulsan desde la adopción de la veda temporal entre abril y junio de cada ańo y las medidas comerciales establecidas.

En este sentido la Nasa Amigable $\mathrm{E}_{1}$ ofrece una alternativa de selectividad que reduce hasta el $31 \%$ las capturas de juveniles y brinda un incremento en las capturas de organismos superiores a los $170 \mathrm{~mm}$ de longitud que alcanzan precios superiores a $\$ 1.00$ (un dólar americano) con respecto a los precios actuales de organismos que oscilan entre el $135 \mathrm{~mm}$ y los $150 \mathrm{~mm}$ de longitud de cola.

Así también ante la reducción de la pesca por buceo hay un aumento del esfuerzo pesquero con nasas, lo cual amerita una alta atención de adopción de la nasa $\mathrm{E}_{1}$, con medidas de control y vigilancia por las autoridades que permitan efectivamente una recuperación de los niveles de la población.

\section{RECOMENDACIONES}

La nasa amigable ha demostrado una reducción significativa en las capturas de juveniles en la pesca artesanal en los cayos miskitus, que supera el $20 \%$ esperado por el proyecto, no obstante, estos resultados solo se limitan a 3 meses de la temporada, por lo cual es conveniente establecer un programa de monitoreo para el inicio de la próxima temporada de pesca 2006 - 2007, considerando que las nasas establecidas se mantendrán en buen estado y vigente su vida útil.

Una reducción en las capturas de juveniles por debajo de la talla legal conduce a afectar el comercio local que principalmente desarrollan las mujeres, por consiguiente es importante brindar alternativas económicas para reducir los efectos en lea economía local de este sector productivo.

Los programas de financiamiento y de fomento económico tanto de las instancias y los gobiernos autónomos y municipales, así como el Instituto de Desarrollo Rural (IDR) debe fomentar el uso de la nasa amigable E1, con el fin de armonizar la sostenibilidad del recurso en la factibilidad biológica y rentabilidad económica.

El esfuerzo de adoptar una nasa amigable E1 debe de sumar la coordinación administrativa entre la municipalidad de Puerto Cabezas, la cual, mediante un proceso de descentralización, le a sido asignado la regulación de la pesquería artesanal, a las autoridades regionales y la fuerza naval para que dirigir esfuerzos en conjunto para adopción por parte de los pescadores artesanales de esta nasa. 


\section{BIBLIOGRAFÍA}

Barnutty. R. (2002). La pesquería de langosta en Nicaragua. AdPesca-MIFIC, Nicaragua.

Barnutty. R. (2006). Informe Nacional de Nicaragua. Taller Regional sobre la Evaluación y Ordenación de la de langosta común del Caribe (Panulirus argus), Mérida, México. MIFIC. ADPESCA CIPA. Nicaragua.

Ehrhardt. N., (2005). Evaluación Bioeconómica del Impacto de la pesca de tallas Ilegales de la Langosta del Mar Caribe, Nicaragua (Informe Final). Pasma-Danida, MIFIC. Managua, Nic. 31p.

Ehrhardt. N., (2006). Estudio Integral sobre la Pesquería de Langosta en el Mar Caribe realizados mediante la técnica de captura de nasa y buceo. Programa Pasma-Danida. 